\title{
Influence of the stacking fault energy and temperature on the prestrain mem- ory effect of face centered cubic metal submitted to cyclic loadings
}

\author{
Clement Keller ${ }^{1, *}$, Gael Marnier ${ }^{2}$, Wilson Veloz ${ }^{1}$, and Lakhdar Taleb ${ }^{1}$ \\ ${ }^{1}$ Groupe de Physique des Matériaux, INSA de Rouen, Université de Rouen, UMR CNRS 6634, avenue de l'Université, 76800 Saint- \\ Etienne du Rouvray, France. \\ ${ }^{2}$ ENSICAEN, bvd du Maréchal Juin, 14020 Caen, France.
}

\begin{abstract}
In this paper, the effect of a monotonic prehardening in tension on the subsequent cyclic stress strain curves is investigated as a function of the deformation mechanisms. To this aim, three materials are employed, copper for wavy dislocation slip, Ni20Cr alloy for planar slip and AISI 316L for intermediate deformation mechanisms. Samples were first prestrained in tension and then submitted to incremental strain cyclic tests at room temperature, and also $200^{\circ} \mathrm{C}$ for the $316 \mathrm{~L}$. Based on the analysis of the flow stress components (backstress and effective stress), the results show that all materials are sensitive to a prehardening depending on the prestrain level and cyclic test characteristics (amplitude and temperature).
\end{abstract}

\section{Introduction}

During the forming processes of metallic materials or accidental service overloads, strong plastic strains are introduced which may influence the following fatigue behavior in service of the metal parts. This link between the prior deformation (called $\mathrm{PH}$ in the following text) sequence and the mechanical behavior in fatigue has been widely studied during the past decades. This $\mathrm{PH}$ sequence generally induces softening depending on the material and the fatigue test.

For FCC materials, several researchers reported that a monotonic or cyclic prehardening sequence generally increases the stress level during the subsequent fatigue cycles compared to the stress obtained without $\mathrm{PH}$, giving rise to a so-called "memory" effect. This effect seems to depend on the stacking fault energy (SFE) of the material and on the maximal strain amplitude. For $\mathrm{Cu}$, it was reported that the flow stress for cycles with a given strain amplitude is not affected by previous cycles with larger strain amplitude [1]. In case of a tensile $\mathrm{PH}$, the memory effect appears if the prestrain is larger than $20 \%$ [2-4]. For stainless steels of low SFE, Murakami et al. [5] described an increase in flow stress in fatigue if the samples were previously submitted to a $\mathrm{PH}$ with larger strain amplitude than during fatigue cycles. However it seems that this effect occurs if the prestrain amplitude is larger than $0.4 \%$. For strong planar material such as $\mathrm{CuAl}$ or $\mathrm{CuZn}$ alloys, the prestrain cannot be recovered and the memory effect seems not to depend on the prior test nor on the subsequent fatigue test $[1,2,4]$. Following all these previous research, the dislocation slip characteristics

*e-mail: clement.keller@insa-rouen.fr clearly control the memory effect.

According to previous results published by the authors, up to three domains of memory effect can appear depending on the material, the prestrain level and cyclic stress amplitude [6]. The objective of this work is to try to explain the origin of these memory domains using experimental characterization of the memory effect for $\mathrm{Cu}, 316 \mathrm{~L}$ and $\mathrm{Ni20Cr}$ with different dislocation slip tendencies with tests performed at room temperature and $200^{\circ} \mathrm{C}$.

\section{Material and experimental procedure}

In order to investigate the role played by the dislocation slip tendency on the memory effect, three materials had been employed : pure copper (wavy slip), AISI 316L (intermediate between wavy and planar slip) and $\mathrm{Ni20Cr}$ alloy (planar slip). The samples of these materials were first submitted to different heat treatments to ensure fully recristallized microstructures with similar grain sizes around $50 \mu \mathrm{m}$ for each material. The ratio of $\frac{\gamma_{\mathrm{sfe}}}{\mu b^{2}}$ which characterizes the planarity of the dislocation slip (planar slip is linked to low value of this parameter) is illustrated for each material on table 1 .

After this first heat treatment, specimens were submitted to a first tensile test. The strain levels of theses tensile tests were determined using the strain hardening stages for each material. For $\mathrm{Cu}, 316 \mathrm{~L}$ and $\mathrm{Ni20Cr}$, three different strain levels in tension were employed, one for each strain hardening stage (namely stages SI, SII, SIII). These stages, obtained usually by a derivative of the tensile 
curve, are illustrated figure 1 for copper. Cyclic behavior of theses samples has been then characterized using incremental strain amplitude test (total strain controlled) in order to obtain the cyclic stress strain curves (CSSC). For each PH strain level, the CSSC is then compared to the one of a virgin specimen. For each strain amplitude of the incremental test, the total number of cycles allows the quasi-saturation of the stress amplitude. Frequency has also been adapted in order to ensure similar strain rate for each strain amplitude. For 316L, in order to check the effect of temperature on the memory effect, similar CSSC are performed at $200^{\circ} \mathrm{C}$ using a three zones lamp furnace.

For the analysis of the mechanical behavior, the stress partition method which decomposes the stress into effective stress and backstress has been employed due to the already characterized link between these two stress components and the deformation mechanisms [7, 8].

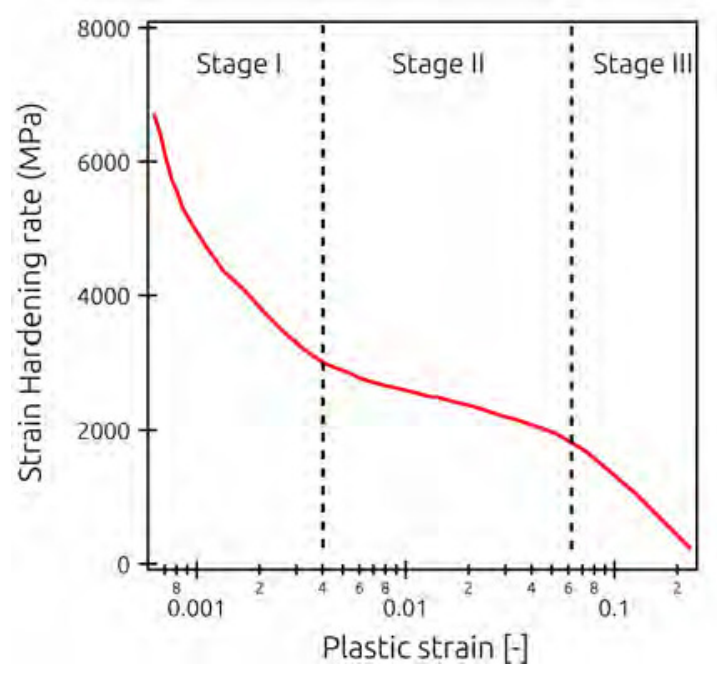

Figure 1. Strain hardening rate as a function of true strain for a polycrystalline copper sample employed in this study.

\section{Results and discussion}

\subsection{Case of copper with wavy slip tendency, at room temperature}

For copper, the figure 2(a) illustrates the CSSC of a virgin (VG) sample and three different prestrained specimens (one for each stage : 0,3\% SI, $2 \%$ SII and $10 \%$ SIII). In agreement with the literature [2,3], cyclic behavior of copper is sensitive to a prehardening depending on various factors. First, the overstress between the prestrain and virgin sample, which characterizes the memory effect, only appears if the prestrain is larger than a critical value. This critical value is related to the transition from the first to the second strain hardening stage in tension, related to the formation of heterogeneous dislocation structures such as tangles or walls [7]. Second, if the plastic strain amplitude of the cyclic test is larger than a second threshold value, then the overstress disapears and the cyclic mechanical behavior no longer depends on the first deformation step. Thus, two domains of memory effect are observed, one with positive overstress, for low cyclic plastic strain amplitude and a second one, without overstress for strain amplitudes larger than a critical value depending on the prestrain level.

Figures 2(b), (c) and (d) represent the evolution, associated with the CSSC of the backstress, the effective stress and mean stress respectively. For the low plastic strain amplitudes, for which the overstress is positive, the value of this parameter is largely linked to the backstress and slighly to the effective stress. Morever, this positive overstress appears simultaneously to a positive mean stress. This mean stress is associated to the backstress and begins to soften for a plastic strain amplitude close to $5 \cdot 10^{-5}$. This value, almost independent on the prestrain level for copper, is in agreement with the value reported by Polak et. al. for which softening occurs for a $\mathrm{Cu}$ virgin sample due to strain localization [9].

This beginning of stress softening for prestrained samples can be also characterized in term of ratio $\sigma^{+} / \sigma^{\mathrm{PH}}$ where $\sigma^{+}$represents the maximal cyclic stress value at which softening begins and $\sigma^{\mathrm{PH}}$ represents the maximal stress value at the end of the prestrain step. For all prestrained copper samples, this ratio is around 0.7 , revealing that softening occurs for stress values lower than the yield stress inherited from the prehardening step. This value is larger than the 0.4 value reported for mild steel [10] and copper [11] both for stress controlled cyclic tests.

\subsection{Case of $316 \mathrm{~L}$ and $\mathrm{Ni20Cr}$ with planar slip tendency}

\subsubsection{At room temperature}

For $316 \mathrm{~L}$ and $\mathrm{Ni20Cr}$ alloys, similar tests as for copper have been performed. Due to the difference in strain hardening stages, prestrained values are different for the three materials. The two memory domains with positive overstress and zero overstress are also characterized for these two materials as illustrated in figure $3(a)$ for $316 \mathrm{~L}$ and 3(b) for Ni20Cr.

Table 1 illustrates the characteristics of the positive overstress domain of memory effect for the three materials and different prestrain levels. In this table is presented, first, the threshold value of plastic strain amplitude, named $\frac{\Delta \varepsilon_{p}}{2}$ soft, which induces the softening of the mean stress (and the overall mechanical behavior). This value is then followed by the associated value of $\sigma^{+} / \sigma^{\mathrm{PH}}$, already defined in the previous section. $\frac{\Delta \varepsilon_{p}}{2}{ }_{\mathrm{P}-0}$ characterizes the plastic strain amplitude corresponding to the transition between the positive and zero overstress and $\Delta \Sigma_{\text {norm }}^{\max }$ represents the maximal flow stress increase for a prestrain sample compared to a virgin one. In order to be compared between the three materials, this value of stress increase 

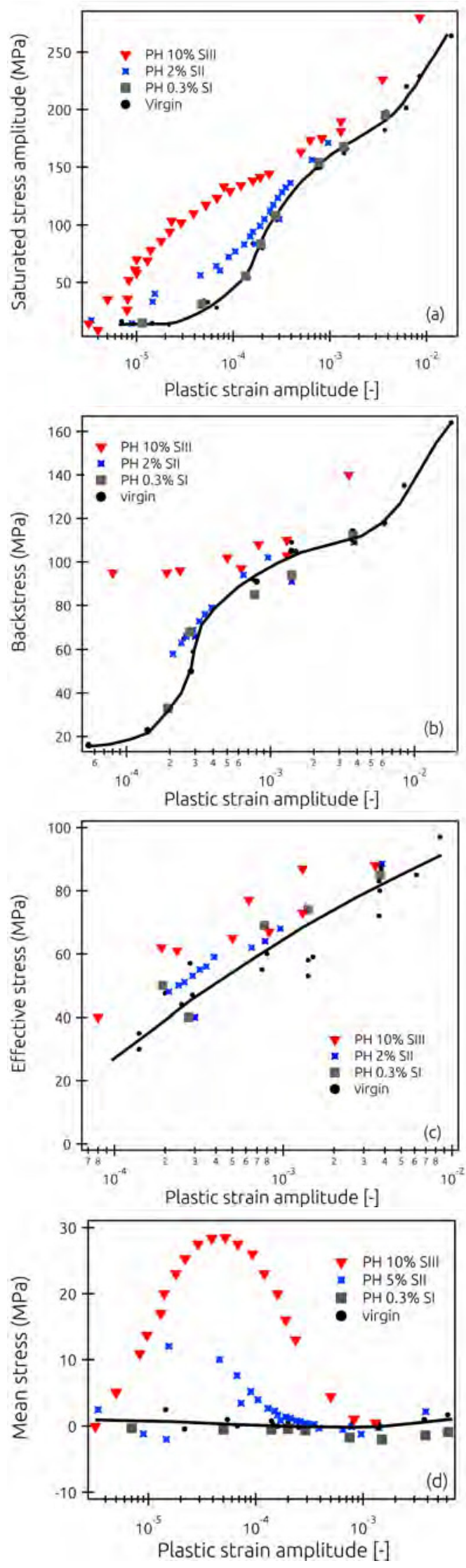

Figure 2. Cyclic stress-strain curves for polycristalline copper submitted to tensile prehardening : (a) saturated stress amplitude, (b) backstress, (c) effective stress and (d) mean stress.

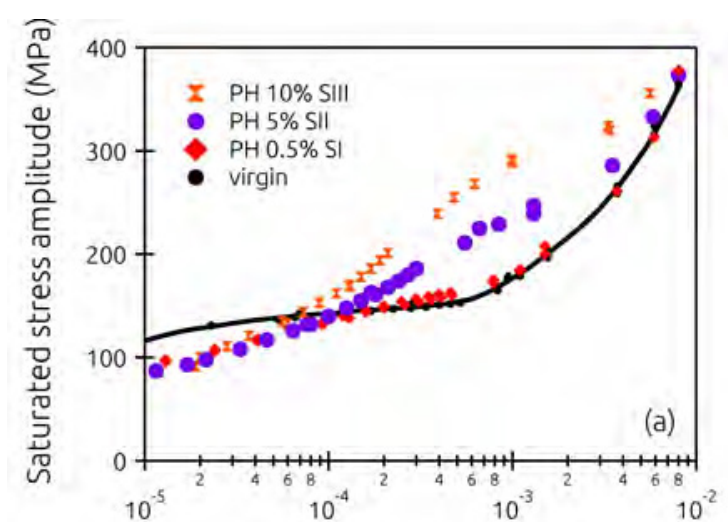

Plastic strain amplitude [-]

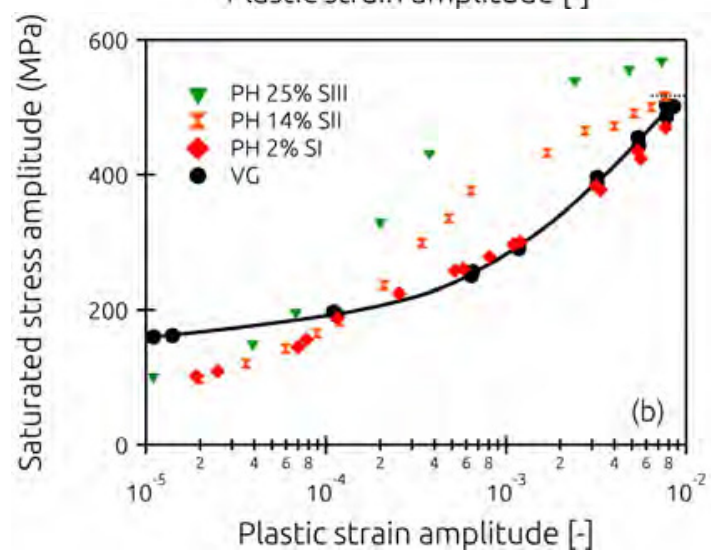

Figure 3. Cyclic stress strain curves for : (a) AISI 316L and (b) Ni20Cr alloy.

is normalised, for each material, by the stress level of the virgin sample (at the strain amplitude involving the maximal increase).

For $316 \mathrm{~L}$ and Ni20Cr, the softening of mean stress starts for larger strain amplitude. Despite large difference in $\frac{\gamma_{\text {sfe }}}{\mu b^{2}}$ between $\mathrm{Ni20Cr}$ and $316 \mathrm{~L}$, the value for these two materials are very close. The comparison of the value of $\sigma^{+} / \sigma^{\mathrm{PH}}$ for the three materials illustrates the influence of the deformation mechanisms of the softening of the mechanical behavior. With the transition from wavy to planar slip, the $\sigma^{+} / \sigma^{\mathrm{PH}}$ ratio progressively shifts towards unity. For Ni20Cr, the softening of the mechanical behavior inherited from the prestrain only occurs if the loading surface is reached whereas, for $316 \mathrm{~L}$ and copper, this softening occurs for maximal stress levels lower than the yield stress.

This evolution clearly highlights the role played by cross slip on the softening and the recovery of the mechanical behavior inherited from the prestrain. In case of $\mathrm{Cu}$, the cross-slip is operative at very low strain amplitude as already characterized in literature [1] whereas for 316L, this cross slip needs larger strain amplitude and stress level to be operative. For Ni20Cr, due to the restricted activity of cross-slip, generalized plasticity is needed to transform the dislocation structures inherited from the monotonous prestrain into those characteristic of cyclic 
Table 1. Summary of the characteristics of the positive overstress domain of memory effect for the three materials and different prestrain levels.

\begin{tabular}{ccccccc} 
Material & $\frac{\gamma_{\text {sfe }}}{\mu b^{2}}\left(10^{6}\right) \mathrm{m}^{-1}$ & Prestrain level & $\frac{\Delta \varepsilon_{p}}{2}{ }_{\text {soft }}$ & $\sigma^{+} / \sigma^{\mathrm{PH}}$ & $\frac{\Delta \varepsilon_{p}}{2}{ }_{\mathrm{P}-0}$ & $\Delta \Sigma_{\text {norm }}^{\max }$ \\
\hline \multirow{2}{*}{$\mathrm{Cu}$} & \multirow{2}{*}{16} & $0.3 \%$ & - & - & - & - \\
& & $2 \%$ & $4.510^{-5}$ & 0.69 & $3.710^{-3}$ & 3.3 \\
& & $10 \%$ & $5.510^{-5}$ & 0.67 & $8.510^{-3}$ & 4.61 \\
\hline \multirow{2}{*}{$316 \mathrm{~L}$} & \multirow{2}{*}{6} & $0.5 \%$ & - & - & - & - \\
& & $5 \%$ & $210^{-4}$ & 0.84 & $810^{-3}$ & 0.4 \\
& & $10 \%$ & $3.910^{-4}$ & 0.845 & $110^{-2}$ & 0.6 \\
\hline \multirow{2}{*}{$\mathrm{Ni} 20 \mathrm{Cr}$} & \multirow{2}{*}{2.5} & $2 \%$ & $210^{-4}$ & 0.99 & $310^{-3}$ & 0.15 \\
& & $14 \%$ & $410^{-4}$ & 0.98 & $8.710^{-3}$ & 0.47 \\
& & $25 \%$ & $510^{-4}$ & 0.98 & $1.310^{-2}$ & 0.83 \\
\hline
\end{tabular}

loadings. The comparison of the last parameter of table 1 between the three materials illustrates the larger strain hardening capacity of the wavy slip material.

Compared to copper, a new domain is also characterized for $316 \mathrm{~L}$ and $\mathrm{Ni20Cr}$ with a negative overstress for the lower plastic strain amplitudes. The analysis of the stress components reveals that this negative overstress is linked to the effective stress. Compared to copper which is significantly textured, $316 \mathrm{~L}$ and Ni20Cr do not, in the unstrained configuration, exhibit any preferential grain orientations. For $316 \mathrm{~L}$ and $\mathrm{Ni} 20 \mathrm{Cr}$, the first strain step involves a grain rotation towards (001) and (111) orientations, the larger the plastic strain, the larger the grain rotation. However, following literature, these two grain orientations involve larger stress amplitude for 316L [12]. As a consequence, this mechanism of grain rotation cannot explain the negative overstress. This overstress is then probably link to the existence, after the prestrain, of forest dislocations which can flip-flop easily for very low plastic strain amplitude during the fatigue test, reducing the stress amplitude [13]. The decrease of the threshold plastic strain amplitude between the negative and positive memory domain for larger prestrain levels in in agreement with this mechanisms. With the increase in monotonic prestrain, dislocations tend to form heterogeneous structures such as walls or cells with large dislocation density which, in turn, reduces their mobility to accommodate the cyclic plastic strain amplitude.

\subsubsection{L at $200^{\circ} \mathrm{C}$}

As illustrated in the previous section, the characteristics of the memory effect strongly depends on the cross-slip activity of the material. For $316 \mathrm{~L}$ with intermediate strain mechanisms between wavy and planar slip, an increase in temperature should increase the cross-slip activity and, in turn, improve the softening capacity of the material. After a first monotonic prestrain at room temperature, the incremental cyclic tests are performed at $200^{\circ} \mathrm{C}$. At this temperature, the ratio $\frac{\gamma_{\text {sfe }}}{\mu b^{2}}$ is very close to the one at Room Temperature - RT - (increase in $\gamma_{\text {sfe }}$ [14] but decrease in $\mu$ [15]) but thermal energy should enhance cross slip activity without dynamic strain ageing.

In order to be compared, all the stress values at RT and $200^{\circ} \mathrm{C}$ were normalized by the shear modulus at each temperature ( $\mu_{\mathrm{RT}}=67 \mathrm{GPa}$ and $\mu_{200}=60.5 \mathrm{GPa}$ [15]). Figure 4 illustrates the normalized stress amplitude as a function of the plastic strain amplitude at RT and $200^{\circ} \mathrm{C}$ for a virgin, a prestrain in tension at $5 \%$ and prestrain in tension at $10 \%$ samples. A signicative difference in stress amplitude value appears between the CSSC of the virgin samples at $\mathrm{RT}$ and $200^{\circ} \mathrm{C}$. This difference is larger at low plastic strain amplitude and seems to be due to the thermal activation of the short range interactions between dislocations and the microstructure.

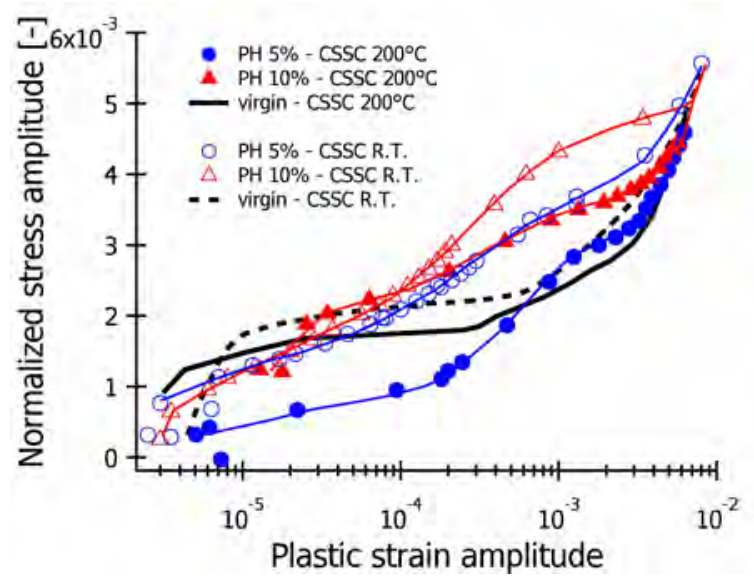

Figure 4. CSSC normalized by the shear modulus obtained at room temperature and $200^{\circ} \mathrm{C}$ for tensile prestrained $316 \mathrm{~L}$ samples.

After a $\mathrm{PH}$ at $5 \%$ in tension, at $200^{\circ} \mathrm{C}$ the range of plastic strain amplitude with positive overstress is considerably reduced. Indeed, for this sample cyclically strained at $200^{\circ} \mathrm{C}$, the transition plastic strain amplitude from negative to positive overstress is increased compared to RT $\left(6.10^{-4}\right.$ at $200^{\circ} \mathrm{C}$ vs $10^{-4}$ at RT) whereas the plastic strain amplitude inducing no memory effect is reduced $\left(4.10^{-4}\right.$ at $200^{\circ} \mathrm{C}$ vs $8.10^{-3}$ at RT). 
Nevertheless, for PH a $10 \%$ in tension, the differences between the CSSC for the two investigated temperatures are weaker. Compared to RT, the elevation in temperature induces a lower plastic strain amplitude value at which the overstress vanished as for PH 5\% and the overstress is significantly reduced as observed in figure 4 .

These results show, first, as expected, that the increase in temperature enhances the crossslip activity which results in a reduction in overstresses and plastic strain amplitude at which this overstress is closed to 0 . Second, the lower effect of the temperature for a prestrain of $10 \%$ compared to $5 \%$ shows that as soon as the third stage of prehardening has been reached, recovery of the dislocation structures inherited from the prehardening step is harder.

\section{Conclusions}

Using three materials $\mathrm{Cu}, 316 \mathrm{~L}$ and $\mathrm{Ni} 20 \mathrm{Cr}$ with different cross slip abilities, the investigation of the prehardening effect on the subsequent cyclic behavior highlights the following features :

- three domains of memory effect can be characterized (negative overstress, positive overstress, no overstress). The negative overstress domain is only observed material with planar slip tendency.

- the transition from positive to 0 overstress domains is linked to mechanical softening. This softening can appear for stress values lower than the yield stress for wavy slip material whereas for planar slip material softening occurs when yield stress is reached.

- an increase in temperature enhances cross slip activity and reduces the plastic strain amplitude range of the cyclic test for which positive overstress is observed.
The memory effect is then triggered by the cross-slip ability of the material which can be affected by the temperature test.

\section{References}

[1] C. Feltner, C. Laird, Acta metallurgica 15, 1621 (1967)

[2] P. Lukáš, M. Klesnil, Materials Science and Engineering 11, 345 (1973)

[3] H.J. Christ, G. Hoffmann, O. Öttinger, Materials Science and Engineering: A 201, 1 (1995)

[4] C. Laird, J. Finney, A. Schwartzman, R. De la Veaux, Journal of Testing and Evaluation 3, 435 (1975)

[5] S. Murakami, M. Kawai, Y. Ohmi, Journal of Engineering materials and Technology 111, 278 (1989)

[6] G. Marnier, C. Keller, L. Taleb, International Journal of Plasticity 78, 64 (2016)

[7] X. Feaugas, H. Haddou, Metallurgical Transactions 34A, 2329 (2003)

[8] C. Keller, E. Hug, X. Feaugas, International Journal of Plasticity 27, 635 (2011)

[9] J. Polák, K. Obrtlík, M. Hájek, A. Vašek, Materials Science and Engineering: A 151, 19 (1992)

[10] H.F. Chai, C. Laird, Materials Science and Engineering 93, 159 (1987)

[11] Q. Fan, H. Chai, M. Tong, Acta Metallurgica Sinica (English letters) 4, 129 (2009)

[12] M. Mineur, P. Villechaise, J. Mendez, Materials Science and Engineering: A 286, 257 (2000)

[13] L. Kunz, P. Lukáš, B. Weiss, D. Melisova, Materials Science and Engineering: A 314, 1 (2001)

[14] L. Rémy, A. Pineau, B. Thomas, Materials Science and Engineering 36, 47 (1978)

[15] M. Fukuhara, A. Sanpei, ISIJ International 33, 508 (1993) 